\title{
Terahertz detection with tunneling quantum dot intersublevel photodetector
}

\author{
X. H. Su, J. Yang, and P. Bhattacharya ${ }^{a}$ \\ Solid State Electronics Laboratory, Department of Electrical Engineering and Computer Science, University \\ of Michigan, Ann Arbor, Michigan 48109-2122 \\ G. Ariyawansa and A. G. U. Perera \\ Department of Physics and Astronomy, Georgia State University, Atlanta, Georgia 30303
}

(Received 17 March 2006; accepted 29 May 2006; published online 21 July 2006)

\begin{abstract}
The characteristics of a tunnel quantum dot intersublevel photodetector, designed for the absorption of terahertz radiation, are described. The absorption region consists of self-organized $\mathrm{In}_{0.6} \mathrm{Al}_{0.4} \mathrm{As} / \mathrm{GaAs}$ quantum dots with tailored electronic properties. Devices exhibit spectral response from 20 to $75 \mu \mathrm{m}(\sim 4 \mathrm{THz})$ with peak at $\sim 50 \mu \mathrm{m}$. The peak responsivity and specific detectivity of the device are $0.45 \mathrm{~A} / \mathrm{W}$ and $10^{8} \mathrm{~cm} \mathrm{~Hz}^{1 / 2} / \mathrm{W}$, respectively, at $4.6 \mathrm{~K}$ for an applied bias of $1 \mathrm{~V}$. Response to terahertz radiation is observed up to $150 \mathrm{~K}$. (C) 2006 American Institute of Physics. [DOI: 10.1063/1.2233808]
\end{abstract}

With the advent of suitable terahertz sources, ${ }^{1,2}$ it is imperative to develop the detection technology in this spectral range. Like any other electromagnetic radiation, terahertz waves can be detected by coherent or incoherent techniques. Most coherent detection schemes utilize frequency conversion. $^{3-5}$ Examples are Schottky diode mixers, nonlinear optical crystals or coatings and gated photoconductive antennas, or switches. Coherent techniques generally provide good sensitivity, but require a high degree of sophistication and instrumentation. The simplest incoherent detectors are heat based, such as bolometers or those made with pyroelectric crystals. These are usually slow and are generally built for low temperature operation. Semiconductor and heterojunction-based schemes that have been characterized include doped Ge detectors, ${ }^{6}$ which provide sensitivity at very low temperatures $(\sim 4 \mathrm{~K})$, photoconductive detectors triggered by femtosecond optical pulses, ${ }^{7}$ heterojunction interfacial work function internal photoemission detectors, ${ }^{8}$ and high-electron mobility transistors operating in the plasma-wave regime. ${ }^{9}$ More recently, quantum-confinement based detectors ${ }^{10}$ have generated interest. These employ heterostructures similar to quantum-well infrared photodetectors (QWIPs) and quantum cascade lasers.

Quantum dot infrared photodetectors (QDIPs), consisting of a multilayered self-organized $\mathrm{In}(\mathrm{Ga}, \mathrm{Al}) \mathrm{As} / \mathrm{Ga}(\mathrm{Al}) \mathrm{As}$ quantum dot active region, have emerged as a technology capable of detecting light across a broad range of infrared (IR) wavelengths. ${ }^{11-14}$ The advantages of QDIPs result from three-dimensional carrier confinement in quantum dots. The associated advantages include (i) intrinsic sensitivity to normal-incidence light, (ii) long lifetime of photoexcited electrons due to reduced electron-phonon scattering, and (iii) low dark current due to three-dimensional quantum confinement and reduced thermionic emission. We recently demonstrated a QDIP in which the dark and photocurrents were decoupled by the incorporation of a double-barrier resonant tunneling heterostructure with each quantum dot layer. The tunnel barrier resonantly transmitted the photoexcited electrons, but blocked most of the dark current composed of electrons with a broad energy distribution. The resulting

${ }^{a)}$ FAX: (734)763-93; electronic mail: pkb@eecs.umich.edu device—the tunnel QDIP (Refs. 15 and 16)—demonstrated far infrared (FIR) operation $\left(\lambda_{\text {peak }}=17 \mu \mathrm{m}\right)$ at room temperature with ultralow dark current. In $\mathrm{In}(\mathrm{Ga}) \mathrm{As} / \mathrm{GaAs}$ quantum dots, the intersublevel energy spacings or the energy difference between the dot and continuum states is normally 40-60 meV, which corresponds to the mid-IR and FIR wavelength ranges. In fact, the longest cutoff wavelength reported for detection with QDIPs is less than $25 \mu \mathrm{m} .{ }^{13}$ Therefore, the dot heterostructure and/or the dot size need to be engineered for detection at longer wavelengths and in the terahertz range. In this letter we report the performance characteristics of tunnel QDIPs, incorporating $\operatorname{In}_{0.6} \mathrm{Al}_{0.4} \mathrm{As} / \mathrm{GaAs}$ self-organized quantum dots of reduced size in the active region, which exhibit spectral response with peak and cutoff wavelengths of 50 and $75 \mu \mathrm{m}(\sim 4.0 \mathrm{THz})$, respectively.

The conduction band diagram of an $\operatorname{In}_{0.6} \mathrm{Al}_{0.4} \mathrm{As} / \mathrm{GaAs}$ quantum dot layer and the associated resonant tunnel heterostructure are shown in Fig. 1(a). A single $60 \AA$ thick $\mathrm{Al}_{0.1} \mathrm{Ga}_{0.9} \mathrm{As}$ barrier is incorporated before each dot layer to form a quantum well with well-defined final states for the photoexcited electrons. The width of the well region and the composition of the barrier can be varied to tune the final states in resonance with the resonant state of the double barrier heterostructure. For detection of terahertz radiation, the energy spacing between the confined state in the dot and the quasibound states in the well has to be of the order of $10 \mathrm{meV}$ or less. This transition is illustrated in Fig. 1(a). To achieve this, we have grown $\operatorname{In}_{0.6} \mathrm{Al}_{0.4} \mathrm{As} / \mathrm{GaAs}$ quantum dots in the active region of the devices, instead of the more conventional InAs dots. Incorporation of $\mathrm{Al}$ into the dot material serves two purposes. First, due to the larger band gap of InAlAs, compared to InAs, the bound state energies are closer to the GaAs barrier energy, and hence to the quasibound states in the well. Second, due to the smaller migration rate of $\mathrm{Al}$ adatoms on the growing surface during epitaxy, the Al-containing islands (dots) are smaller in size compared to InAs dots and the dot confined states are higher in energy. In this study, the dot size in the devices was also varied, by the growth parameters, to tune the absorption frequency. Finally, the density of $\operatorname{In}_{0.6} \mathrm{Al}_{0.4} \mathrm{As}$ dots $(\sim 3$ $\times 10^{11} \mathrm{~cm}^{-2}$ ) is generally an order of magnitude larger than 

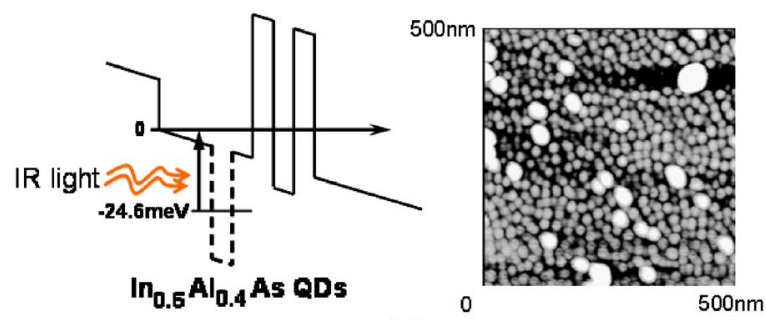

(a)

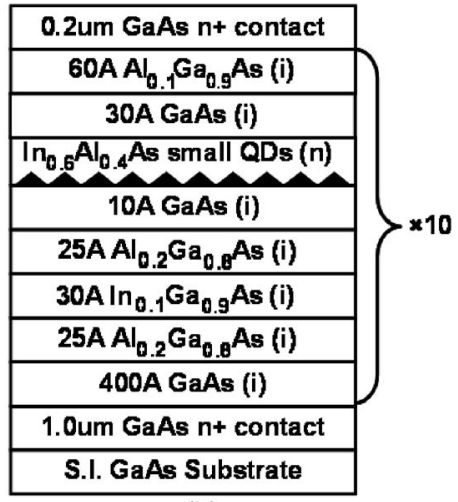

(b)

FIG. 1. (a) Single period conduction band schematic diagram and AFM image of $\operatorname{In}_{0.6} \mathrm{Al}_{0.4} \mathrm{As} / \mathrm{GaAs}$ dots; (b) schematic heterostructure of T-QDIP grown by molecular beam epitaxy.

that of InAs dots, which helps to absorb more of the incident radiation.

The average size of the $\operatorname{In}_{0.6} \mathrm{Al}_{0.4} \mathrm{As}$ dots was estimated from atomic force microscopy (AFM) measurements. An AFM image of an ensemble of the smaller sized dots is also shown in Fig. 1(a). The base and height of the nearpyramidal dots are $\sim 140$ and $\sim 45 \AA$, respectively. An eightband $\mathbf{k} \cdot \mathbf{p}$ model $^{17}$ is used to calculate the electronic states in the quantum dots, taking into account the strain in the dots with the valence force field model and the size mentioned above. The quasibound states in the well are obtained from a one-dimensional solution of the Schrödinger equation. The energies of the bound states are indicated in Fig. 1(a).

The tunneling QDIP heterostructures were grown by molecular beam epitaxy in an EPI Mod Gen II system equipped with an arsenic cracker. The complete device heterostructure, grown on (001)-oriented semi-insulating GaAs substrate, is schematically shown in Fig. 1(b). The GaAs and AlGaAs layers were grown at $610{ }^{\circ} \mathrm{C}$ and the rest of the heterostructure was grown at $500{ }^{\circ} \mathrm{C}$. The top and bottom GaAs contact layers are doped $n$-type with $\mathrm{Si}$ to a level of $2 \times 10^{18} \mathrm{~cm}^{-3}$. The quantum dots are also doped with Si such that the bound states are occupied. Mesa-shaped vertical $n-i-n$ devices for top illumination were fabricated by standard photolithography, wet chemical etching, and contact metallization techniques. The top and bottom $n$-type contacts were formed by evaporated $\mathrm{Ni} / \mathrm{Ge} / \mathrm{Au} / \mathrm{Ti} / \mathrm{Au}=250 / 325 / 650 / 200 / 2000 \AA$ and then annealed. The radius of the illuminated area inside the top ring contact is $300 \mu \mathrm{m}$. For measurements, the devices are mounted onto a chip carrier with silver epoxy and individual devices are wire bonded to separate leads of the carrier. The chip carrier is then inserted into a liquid helium dewar equipped with special windows which are transparent to very long-wavelength light $(10-80 \mu \mathrm{m})$.

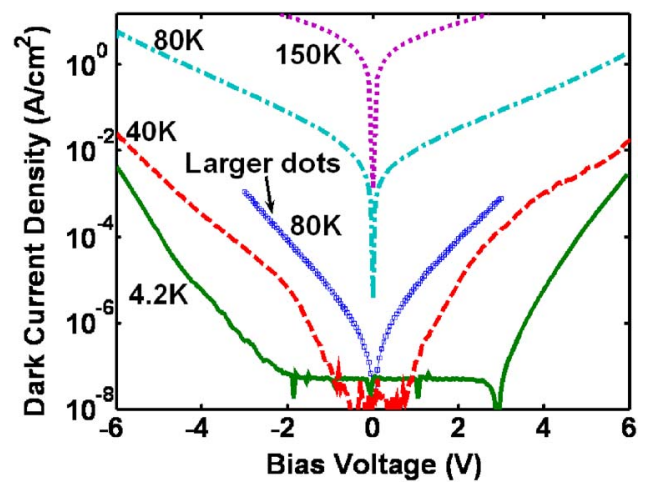

FIG. 2. Measured dark current density as a function of bias and temperature.

The dark current density of the device, with the smaller sized InAlAs dots as a function of bias voltage and temperature, shown in Fig. 2, is measured with a HP 4145 semiconductor parameter analyzer. The dark current densities at a bias of $1 \mathrm{~V}$ are $4.77 \times 10^{-8}, 2.03 \times 10^{-2}$, and $4.09 \mathrm{~A} / \mathrm{cm}^{2}$ at $4.2,80$, and $150 \mathrm{~K}$, respectively. These values are very low compared to other terahertz detectors. ${ }^{8,18}$ We believe this is due to the existence of the double barrier tunnel heterostructure. For comparison, the dark current densities in a device with larger sized dots, measured at $80 \mathrm{~K}$, are also included. It is apparent that devices with larger dots are more suitable for high temperature operation.

The spectral response of the devices is measured with a BOMEM MB-155 Fourier transform infrared (FTIR) spectrophotometer and a globar broadband source, under normal incidence. For calibration of the results, the spectral response of a composite bolometer, with a known sensitivity, is also measured with identical optical elements and optical path. The calibrated spectral response of the T-QDIP with smaller dots at $4.6 \mathrm{~K}$, with bias of $1.0 \mathrm{~V}$, is shown Fig. 3(a). The peak responsivity is about $0.45 \mathrm{~A} / \mathrm{W}$ and the wavelength corresponding to this peak is around $50 \mu \mathrm{m}$ which agrees with the calculated energy difference between the QD bound state and the quasibound state in the well of $24.6 \mathrm{meV}$ $(50.4 \mu \mathrm{m})$. The cutoff wavelength is $\sim 75 \mu \mathrm{m}$, which corresponds to $\sim 4.0 \mathrm{THz}$. The transition between the dot state and the state in the well is expected to be sensitive to normal incidence or $s$-polarized radiation. This has been verified earlier in QDIPs. ${ }^{13}$ In the dot-well system, the states in the well are no longer $z$ confined, but also have a radial component. The dark region (dip) in the spectral response centered at $\sim 36 \mu \mathrm{m}$ is due to longitudinal optical phonon absorption in GaAs. This same phenomenon has been observed by other GaAs based detectors. ${ }^{8,18}$ The spectral response appears to be fairly broad. The transition is believed to be from the dot bound states to quasibound states in the well and the spectral width of such transitions will not match the observed full width at half maximum (FWHM) of $\sim 35 \mu \mathrm{m}$, which corresponds to $23 \mathrm{meV}$. We attribute the observed linewidth to size nonuniformity of the self-organized dots which give rise to linewidths of $\sim 30-40 \mathrm{meV}$ in the interband photoluminescence spectra. Figures 3(b) and 3(c) show responsivity spectra at higher temperatures from a device with the larger sized $\mathrm{In}_{0.6} \mathrm{Al}_{0.4} \mathrm{As}$ dots. The long-wavelength response is shifted to shorter wavelengths. The device can be operated at a temperature of $150 \mathrm{~K}$, which is high compared to other photon-based terahertz detectors. In order to achieve 1-3 THz operation at reasonably high temperature the dot 

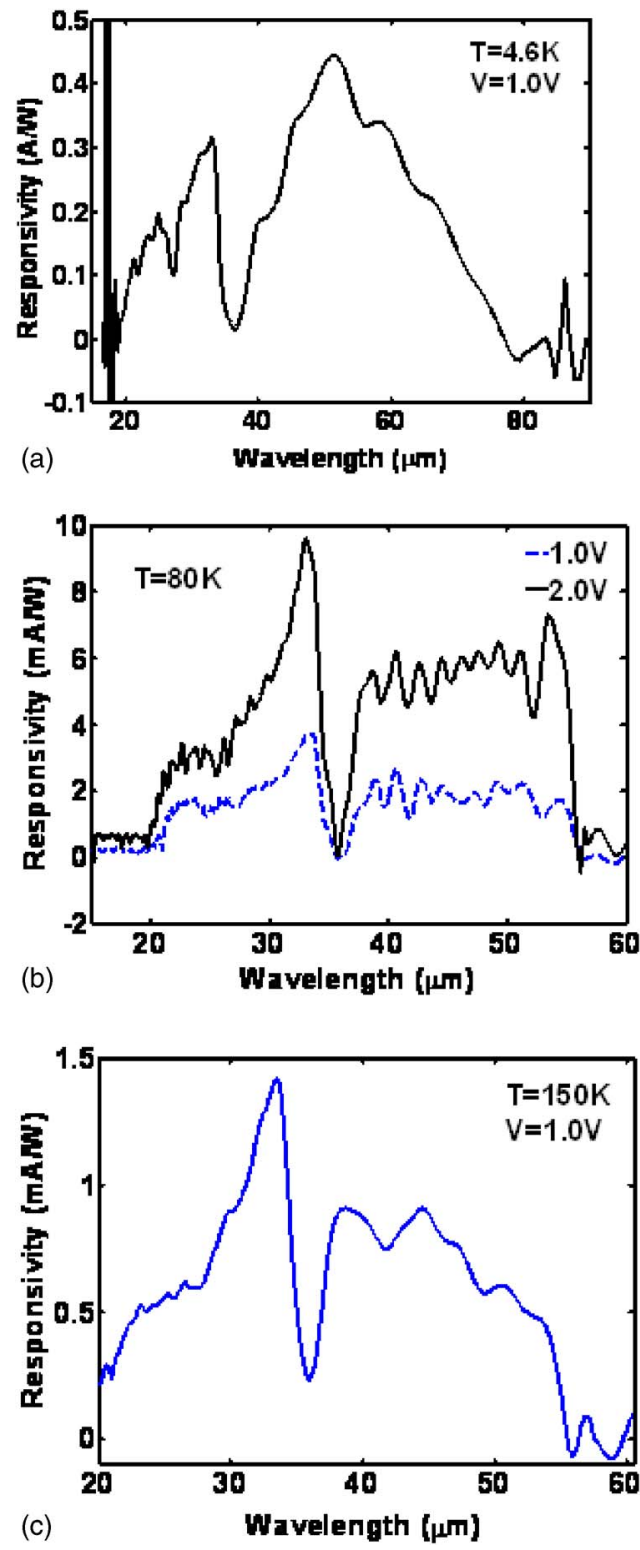

FIG. 3. Measured spectral responsivity of T-QDIP at (a) $4.6 \mathrm{~K}$, (b) $80 \mathrm{~K}$, and (c) $150 \mathrm{~K}$ under bias of $1 \mathrm{~V}$.

size needs to be reduced, the size uniformity improved, and the tunnel heterostructure needs to be further optimized to keep the dark current low. The dot size can be reduced by increasing the $\mathrm{Al}$ content in the dots and by reducing the growth temperature. We have recently reported a room temperature photoluminescence linewidth of $17 \mathrm{meV}$ in InAs quantum dots, ${ }^{19}$ which is limited by homogeneous broadening. All these aspects are currently being investigated.

The specific detectivity $\left(D^{*}\right)$ of the devices at different temperatures and applied biases is obtained from the peak responsivity $R_{p}$ and noise density spectra $S_{i}$. The latter is measured with a dual channel fast Fourier transform (FFT) signal analyzer, which displays a FFT spectrum of voltage versus frequency, and a low noise preamplifier. A thick copper plate is used as a radiation shield to provide the dark conditions for the measurements. The value of $D^{*}$ is calculated from

$$
D^{*}=R_{p} A^{1 / 2} / S_{i}^{1 / 2} \quad\left(\mathrm{~cm} \mathrm{~Hz}^{1 / 2} / \mathrm{W}\right),
$$

where $A$ is the illuminated area of the detector. The measured $D^{*}$ values are $1.64 \times 10^{8}$ and $4.98 \times 10^{7} \mathrm{~cm} \mathrm{~Hz}^{1 / 2} / \mathrm{W}$ at 4.6 and $80 \mathrm{~K}$, respectively, under a bias of $1 \mathrm{~V}$.

In conclusion, we report the detection of normally incident terahertz radiation with a tunneling QDIP. One of the devices exhibits a maximum spectral response peak at $\sim 50 \mu \mathrm{m}$ and cutoff at $\sim 75 \mu \mathrm{m}$ with a specific detectivity of $1.64 \times 10^{8} \mathrm{~cm} \mathrm{~Hz}^{1 / 2} / \mathrm{W}$ and peak responsivity of $0.45 \mathrm{~A} / \mathrm{W}$ at $4.6 \mathrm{~K}$. A peak responsivity of $1.2 \mathrm{~mA} / \mathrm{W}$ is measured in another device at $150 \mathrm{~K}$.

This work at the University of Michigan is supported by the Army Research Office (MURI program) under Grant No. DAAD19-01-1-0462 and at Georgia State University by the National Science Foundation under Grant No. ECS-0140434.

${ }^{1}$ A. L. Betz, R. T. Boreiko, B. S. Williams, S. Kumar, Q. Hu, and J. L. Reno, Opt. Lett. 30, 1837 (2005).

${ }^{2}$ S. Verghese, K. A. McIntosh, and E. R. Brown, IEEE Trans. Microwave Theory Tech. 45, 1301 (1997).

${ }^{3}$ D. H. Auston, K. P. Cheung, and P. R. Smith, Appl. Phys. Lett. 45, 284 (1984).

${ }^{4}$ I. Brener, D. Dykaar, A. Frommer, L. N. Pfeiffer, J. Lopata, J. Wynn, K. West, and M. C. Nuss, Opt. Lett. 21, 1924 (1996).

${ }^{5}$ Y. Cai, I. Brener, J. Lopata, J. Wynn, L. Pfeiffer, and J. Federici, Appl. Phys. Lett. 71, 2076 (1997).

${ }^{6}$ E. E. Haller, Infrared Phys. 35, 127 (1994).

${ }^{7}$ M. Suzuki and M. Tonouchi, Appl. Phys. Lett. 86, 163504 (2005).

${ }^{8}$ M. B. M. Rinzan, A. G. U. Perera, S. G. Matsik, H. C. Liu, Z. Wasilewski, and M. Buchanan, Appl. Phys. Lett. 86, 071112 (2005).

${ }^{9}$ J. Lü and M. S. Shur, Appl. Phys. Lett. 78, 2587 (2001).

${ }^{10}$ H. C. Liu, C. Y. Song, A. J. SpringThorpe, and J. C. Cao, Appl. Phys. Lett. 84, 4068 (2004)

${ }^{11}$ J. Phillips, P. Bhattacharya, S. W. Kennerly, D. W. Beekman, and M. Dutta, IEEE J. Quantum Electron. 35, 936 (1999).

${ }^{12}$ E. Kim, A. Madhukar, Z. Ye, and J. C. Campbell, Appl. Phys. Lett. 84, 3277 (2004)

${ }^{13}$ S. Krishna, S. Raghavan, G. von Winckel, A. Stintz, G. Ariyawansa, S. G. Matsik, and A. G. U. Perera, Appl. Phys. Lett. 83, 2745 (2003).

${ }^{14}$ S. Chakrabarti, A. D. Stiff-Roberts, P. Bhattacharya, S. D. Gunapala, S. Bandara, S. B. Rafol, and S. W. Kennerly, IEEE Photonics Technol. Lett. 16, 1361 (2004).

${ }^{15}$ P. Bhattacharya, X. H. Su, S. Chakrabarti, G. Ariyawansa, and A. G. U. Perera, Appl. Phys. Lett. 86, 191106 (2005).

${ }^{16}$ X. H. Su, S. Chakrabarti, P. Bhattacharya, G. Ariyawansa, and A. G. U. Perera, IEEE J. Quantum Electron. 41, 974 (2005).

${ }^{17}$ H. Jiang and J. Singh, Phys. Rev. B 56, 4696 (1998).

${ }^{18}$ D. G. Esaev, M. B. M. Rinzan, S. G. Matsik, A. G. U. Perera, H. C. Liu, B. N. Zvonkov, V. I. Gavrilenko, and A. A. Belyanin, J. Appl. Phys. 95, 512 (2004).

${ }^{19}$ Z. Mi and P. Bhattacharya, J. Appl. Phys. 98, 023510 (2005). 